\title{
Ensuring Exclusive Human Milk Diet for All Babies in COVID-19 Times
}

\author{
Ruchika Chugh SaChdeva ${ }^{1}$, Suksham Jain ${ }^{2}$, Suchandra MukherJeE ${ }^{3}$ And Jai Singh ${ }^{4}$ \\ From ${ }^{1}$ Maternal, Newborn, Child Health and Nutrition, PATH, New Delhi, ${ }^{2}$ Department of Neonatology, Government Medical \\ College and Hospital, Chandigarh; ${ }^{3}$ Department of Neonatology, Institute of Post-Graduate Medical Education and Research and \\ Seth Sukhlal Karnani Memorial Hospital, Kolkata, West Bengal; and ${ }^{4}$ Department of Neonatology, District Hospital Chittorgarh, \\ Rajasthan; India. \\ Correspondence to: Ruchika Chugh Sachdeva, Deputy Director, Maternal, Newborn, Child Health and Nutrition, PATH 15th Floor, \\ Gopal Das Bhawan, Barakhamba Road New Delhi-110001, India.rsachdeva@path.org \\ Published online: June 09, 2020: PII: S097475591600191
}

The coronavirus disease (COVID-19) pandemic has ramifications for the delivery of newborn nutrition and care services. World Health Organization recommends continuation of breastfeeding in these difficult times, with due precautions. If direct breastfeeding is not possible, milk expression should be explored. Pasteurized donor human milk from milk banks may be fed if mother's own milk is not available. To universalize access to human milk, the Indian government has proposed the establishment of comprehensive lactation management centers/milk banks, lactation management units, and lactation support units at all levels of the public health system. Due to COVID-19, these centers are encountering additional challenges cutting across interventions of rooming in, breastfeeding, milk expression, and provision of donor milk and kangaroo mother care. We discuss issues faced and alleviation measures taken by these centres in relation to provision of an exclusive human milk diet for infants during the pandemic.

Keywords: Breastfeeding, Donor Human Milk, Kangaroo Mother Care, Pandemic.

Published online: June 09, 2020; PII: S097475591600191

$\mathrm{T}$ he severe acute respiratory syndrome coronavirus 2 (SARS-CoV-2) pandemic has brought forth unprecedented global and local challenges, more so in developing countries like India. While on one hand, the country has launched measures including a nationwide lockdown to curb the spread of the disease, on the other, efforts are on to minimize disruptions to the delivery of essential services such as maternal and newborn health and nutrition [1]. Estimates suggest that lower coverage of interventions for six months due to the pandemic will result in $17 \%$ (1.15 million) more newborn and child deaths globally [2].

Breastfeeding and a human milk diet has a key role in preventing life-threatening infections in infants during the COVID-19 pandemic thus reducing the stress on the health system. Considering that, India has a high newborn mortality rate and a third of all preterm births, in the world [3], ensuring adequate availability of breastmilk becomes even more pertinent. Of the 25 million births, nearly $43 \%$ (11 Million) are not breastfed within the first hour [4] and nearly 30-50\% preterm/sick babies in neonatal intensive care units (NICU) in India lack access to breastmilk [5].

\section{INFANT FEEDING AND COVID-19}

So far, SARS-CoV-2 has not been detected in the breastmilk of mothers with COVID-19 [6]. Preliminary data indicates a strong immunoglobulin Adominant SARSCoV-2 immune response in breast milk of COVID-19 infected mothers [7]. In February 2020, the World Health Organization (WHO) advised early and exclusive breastfeeding for COVID-19 suspected and confirmed mothers, while encouraging them to take adequate precautions. Mothers who are too ill to breastfeed are advised to feed their expressed breastmilk [8]. Guidelines by Indian Council of Medical Research (ICMR) and other professional bodies propagate similar advice [9-11].

In absence of the above, pasteurized donor human milk (PDHM) from a human milk bank (HMB) is recommended over formula milk. Once mothers recover, they should be supported for relactation [8,11]. PDHM compared to formula milk reduces the risk of sepsis, necrotizing enterocolitis, diarrhea and feeding intolerance, and the length of stay in NICU [12]. Feeding supplementary PDHM is associated with increased exclusive breastfeeding at six months of life [13].

As per the National Guidelines for Lactation Management in Public Health Facilities [14], it is proposed to establish facility-based lactation management centers at all levels of the public health system. Comprehensive lactation management centers (CLMCs) or integrated milk banks at tertiary centers, lactation management units at secondary centers, and 
lactation support units at primary care centers [14]. Currently, 83 CLMCs exist in India, yet the number is insufficient to meet the large demand for human milk, despite 1,000,35 babies having benefitted from PDHM in the year 2019 (data not shown).

\section{ENSURING ACCESS TO HUMAN MILK}

The COVID-19 pandemic has posed several challenges to the provision of newborn nutrition and care interventions including maternal support, breastfeeding, kangaroo mother care (KMC), family participatory care, and human milk bank operations and supply of PDHM. A comprehensive newborn nutrition and care response comprising of optimal lactation support and accessibility to PDHM is needed to improve neonatal health outcomes. Globally, efforts are on to address such challenges. Recently, a virtual group consisting of members from 37 countries has been formed to facilitate the sharing of information and best practices and discussion of evidence to prepare human milk banks to deal with COVID-19 challenges [15].

We herein highlight the issues related to access to newborn nutrition and care and how facilities in the country are addressing them during and beyond COVID times.

\section{Access to Mothers' Own Milk}

Suspension of outpatient services and restrictions on travel, in addition to staff shortage (absence and irregularity due to travel restrictions in lockdown), made it difficult for mothers and families to get access to feeding support. Mothers are being discharged sooner and if their babies are in neonatal intensive care units, they get little opportunity to learn to breastfeed or express and feed mothers' milk to their babies due to which, they are resorting to formula feeding at homes. Due to infrastructure constraints and mandate to transfer COVID-19 positive mothers to separate COVID-19 wards, many facilities are separating COVID-19 positive mothers from their babies, making breastfeeding a challenge. Few facilities that can isolate, place mother and baby together, in such cases, breastfeeding is practiced following strict hygiene measures. Early discharge of the baby along with a healthy attendant is encouraged at some places, which makes breastfeeding difficult. However, some hospitals prefer not to discharge babies, owing to fears that family may have COVID-19 cases. There may be hesitation in allowing mothers of outborn neonates to breastfeed, express milk, or conduct KMC (as they are not sure of their background) as an infection control measure. Some facilities end up feeding PDHM or formula owing to a shortage of staff to handle the collection and transfer of expressed mother's milk. Mothers and families, and health care workers have fear and confusion around COVID-19 and breastfeeding, along with a sense of heightened stigma about the disease which is impacting access to breastmilk.

Guidance suggests hospitals should provide more information, psychosocial, and technical support to lactating mothers and their families to build their confidence and establish and sustain milk supply. Providers or family members (with personal protection equipment) should provide support in breastfeeding and caring for newborns [11]. Facilities have reported adopting various methods to improve lactation in mothers, including introducing music and religious book reading sessions to make mothers feel relaxed; allocating rooms in NICUs for mothers along with regular thermal screening and adherence to strict hygiene measures; arranging for additional breast pumps for all mothers; and information dissemination platforms through calls, live chats, and dedicated helplines. Some centers are using these to provide support and guidance on the continuum of breastfeeding.

\section{Rooming-in and KMC}

WHO and the Italian Society for Neonatology recommends that infants and mothers with suspected or confirmed COVID-19 should be allowed to remain together and practice rooming-in throughout the day and night $[16,17]$. Some facilities temporarily discontinued the practice of $\mathrm{KMC}$ for all eligible neonates as a precaution but resumed it when babies showed signs of distress (personal communication - Dr Kumutha J, Chennai). Hospitals, in general, do not recommend $\mathrm{KMC}$ by COVID-19 positive mothers, and recommend engaging healthy family members to conduct KMC. Many staff and mothers are not comfortable in practicing $\mathrm{KMC}$ for fear of transmission of infection via close contact. Rooming-in is difficult as COVID-19 positive mothers are shifted to COVID-19 wards/ facilities while their babies remain in the neonatal units.

Guidance by professional bodies indicates that facilities where isolation of COVID-19 suspected/ confirmed mother and her baby is possible, rooming-in with direct breastfeeding should take place. Where isolation is not possible, mother should be temporarily separated from baby until she is confirmed negative [11]. Some facilities have created separate COVID postnatal wards wherein babies are bedded in with mothers. Lactation counsellors use microphone to conduct group counselling maintaining adequate distance.

\section{Donor Human Milk}

PDHM is a lifesaving resource for babies in neonatal intensive care units who cannot receive their mother's milk 
[18]. Various studies have shown thermal inactivation (specifically heat treatment of $60{ }^{\circ} \mathrm{C}$ for 30 minutes of donor human milk) of respiratory viruses particularly the MERS corona virus [19,20]. A recent study regarding heat stability of SARS-CoV-2 suggests that it is killed at $56^{\circ} \mathrm{C}$ centigrade within 30 minutes [21]. Chambers, et al. [22] also demonstrated that when control breastmilk samples spiked with replication competent SARS-CoV-2 virus were treated by Holder pasteurization, no replication-competent virus or viral RNA was detectable. Another recent study conducted on five different SARS-CoV-2 isolates from Germany, France and the Netherlands in five individual breast milk samples showed that human breast milk containing infectious SARS-CoV-2 can be efficiently inactivated using standard holder pasteurization [23]. Holder pasteurization used in HMBs exposes the milk to $62.5^{\circ} \mathrm{C}$ for 30 minutes.

Many centers have temporarily suspended human milk banking services for reasons such as absenteeism and irregularity of staff due to imposed lockdown. Number of facility-based milk donors has reduced because of earlier discharges and discontinuation of outpatient services. Amid lockdown, home collection of donor milk, and community milk collection has almost stopped. Some mothers may be reluctant to donate fearing donation as a point of exposure. Few hospitals are finding it hard to devote time to milk culture and outsourcing has become difficult for others due to transportation restrictions. To make up for the reduced supply, some units are rationing PDHM to the most vulnerable, small, and sick babies, which may lead to increase in formula feeding for babies without access to maternal milk.

PDHM provided during any COVID-19 related separation, should be coupled with optimal lactation support to ensure maternal milk supply at the earliest. This reduces the excess demand for PDHM which can be given to those who need it the most [15]. Guidelines by professional bodies suggest greater vigilance and modification of donor screening procedures [11]. CLMC staff are asking additional questions related to symptoms, travel and contact history, and excluding symptomatic / at-risk donors. Strict hygiene procedures are followed while supporting expression, transportation, and handling of milk. Despite restrictions related to external lab facilities, few CLMCs are collecting and storing donor milk so that it can be tested once lab facilities become available. One facility has created a WhatsApp group of lactating mothers to motivate them to donate milk and is picking up donated milk from homes, which has led to an increase in donation and number of babies benefitting from donor milk [Personal communication: Dr Senthil Kumar, Coimbatore].

\section{CONCLUSIONS}

Strengthening of lactation management systems to universalize access to human milk is a key strategy to reduce newborn morbidity and mortality during and beyond the pandemic. We, therefore, call on partners and stakeholders to highlight importance of neonatal nutrition during the pandemic; fund research on breastmilk and COVID-19; facilitate innovations across CLMC processes to improve access to maternal milk and PDHM; equip zonal reference centres to support facilities to adequately prepare for future emergencies; and support the consolidation of the countrywide Human Milk Bank Association of India to facilitate communication, sharing of data and best practices, and evidence generation among milk banks. Now is the time for all stakeholders to jointly mount a comprehensive response against the virus - one that encompasses effective newborn nutrition and care practices to protect the future citizens of our country.

Acknowledgments: Dr. Kumutha J, Saveetha Medical College, Chennai, and Expert Advisor, National Health Mission -Tamil Nadu; Dr. S. Sitaraman, Sawai Man Singh Medical College, Jaipur; Dr. Poonam Singh, Surat Municipal Institute of Medical Education \& Research, Surat; Dr. Jayashree Mondkar, Lokmanya Tilak Medical College and Hospital, Mumbai; Dr. Mala Kumar, King George Medical University and Hospital, Lucknow; Dr. Kajal Jain, All India Institute of Medical Science, New Delhi; Dr. Sushma Nangia, Lady Hardinge Medical College and Hospital, New Delhi; Dr. Raghuram Mallaiah and Ohika Chakravarty, Fortis La Femme, New Delhi for providing critical information on neonatal feeding practices during COVID-19 pandemic. Gillian Weaver and Natalie Shenker, global human milk bank specialists for their inputs on the design and Dr Cyril Engmann, Global Program Leader Maternal, Newborn, Child Health and Nutrition Program, Dr Kiersten Israel-Ballard, Associate Director - Maternal, Newborn and Child Health and Nutrition and Dr. Sudhir Maknikar, Director Programs-RMNCHA+N, PATH for their inputs to the manuscript. Dr. Jayendra Kasar, Technical Specialist, Centre for Health Research and Innovation-CHRI for compiling feeding data from various facilities and providing valuable inputs to the manuscript. Paramita Kundu, Consultant, CHRI for her support in writing and editing this manuscript. Contributors: RCS: conceptualized the design, contributed to manuscript writing and revising it and provided final approval of the manuscript; SJ: provided technical guidance on the content, reviewed the manuscript, and provided final approval of the manuscript. SM: provided technical inputs on the content, reviewed, and provided final approval of the manuscript; JS: provided inputs on the content, reviewed the manuscript, and provided final approval of the manuscript.

Funding: None; Competing interest: None stated.

\section{REFERENCES}

1. Enabling Delivery of Essential Health Services during the 
COVID 19 Outbreak: Guidance note. Available from: https://www.mohfw.gov.in/pdf/Essential services during COVID19 updated 0411201.pdf. Accessed April 27, 2020.

2. Roberton T, Carter ED, Chou VB, Stegmuller AR, Jackson B D, Yvonne T, et al. Early estimates of the indirect effects of the COVID-19 pandemic on maternal and child mortality in low-income and middle-income countries: A modelling study. Lancet Glob Health. 2020. Available from: https://www.thelancet.com/journals/langlo/article/ PIIS2214-109X(20)30229-1/fulltext. Accessed May 13, 2020.

3. Sankar MJ, Neogi SB, Sharma J, Chauhan M, Srivastava R, Prabhakar PK, et al. State of newborn health in India. J Perinatol. 2016;36:S3-S8.

4. Ministry of Health and Family Welfare (MoHFW), Government of India, UNICEF and Population Council. 2019. Comprehensive National Nutrition Survey (CNNS) National Report, New Delhi.

5. Sachdeva RC, Mondkar J, Shanbhag S, Sinha M, Khan A, Dasgupta R. A landscape analysis of human milk banks in India. Indian Pediatr. 2019:56:663-68.

6. Chen H, Guo J, Wang C, Luo F, Yu X, Zhang W, et al. Clinical characteristics and intrauterine vertical transmission potential of COVID-19 infection in nine pregnant women: A retrospective review of medical records. Lancet. 2020;395:809-15.

7. Fox A, Marino J, Amanat F, Krammer F, Hahn-Holbrook $\mathrm{J}$, Zolla-Pazner S, et al. Evidence of a significant secretory-IgA-dominant SARS-CoV-2 immune response in human milk following recovery from COVID-19 8 medRxiv. 2020. [Pre-print].

8. WHO. 2020 Breastfeeding advice during the COVID-19 outbreak. Available from: http://www.emro.who.int/ nutrition/nutrition-infocus/breastfeeding-advice-duringCOVID-19-outbreak.html. Accessed April 23, 2020.

9. ICMR - National Institute for Research in Reproductive Health. 2020. Guidance for Management of Pregnant Women in COVID-19 Pandemic. Available from: https:// icmr.nic.in/sites/default/files/upload_documents/ Guidance_for_Management_of_P regnant_Women_in_ COVID19_Pandemic_12042020.pdf. Accessed April 27, 2020.

10. Chawla D, Chirla D, Dalwai S, Deorari AK, Ganatra A, Gandhi A, et al. Perinatal-Neonatal Management of COVID-19 Infection - Guidelines of the Federation of Obstetric and Gynecological Societies of India (FOGSI), National Neonatology Forum of India (NNF), and Indian Academy of Pediatrics (IAP). Indian Pediatr. 2020 April 1 [Online ahead of print]. Available from: https:// www.indianpediatrics.net/COVID01.04.2020/RECC00154.pdf. Accessed April 28, 2020.

11. The Human Milk Bank Association of India, IAP-IYCF Chapter, PATH April 2020. Guidance for use of Human Milk in the context of COVID-19 in India Ver. 1.0. Available from: https://www.path.org/resources/guidanceuse-human-milk-india-context-covid-19/. Accessed April
28, 2020 .

12. Quigley M, Embleton ND, McGuire W. Formula versus donor breast milk for feeding preterm or low birth weight infants. Cochrane Database Syst Rev. 2019;6: CD002971.

13. Merjaneh N, Williams P, Inman S, Schumacher M, Ciurte A, Smotherman C, et al. The impact on the exclusive breastfeeding rate at 6 months of life of introducing supplementary donor milk into the level 1 newborn nursery. J Perinatol. 2020 Mar 30. [Epub ahead of print] Available from https://pubmed.ncbi.nlm.nih.gov/ 32231257. Accessed April 28,2020

14. National Guidelines on Lactation Management Centres in Public Health Facilities. Child Health Division, Ministry of Health and Family Welfare, Government of India. Available from: http://nhm.gov.in/images/pdf/ programmes/IYCF/National_Guidelines_Lactation_ Management_Centres.pdf. Accessed April 27, 2020.

15. Shenkar N, on behalf of Virtual Collaborative Network of Human milk Banks and Associations Maintaining safety and service provision in human milk banking: A call to action in response to the COVID-19 pandemic. Lancet Child Adolesc Health. 2020;S2352-4642(20)30134-6. Available from: https://www.thelancet.com/journals/ lanchi/article/PIIS2352-4642(20)30134-6/fulltext. Accessed May 11, 2020.

16. WHO, 13 March, 2020, Clinical Management of severe acute respiratory infection (SARI) when COVID-19 disease is suspected. Interim Guidance. Available from: https://www.who.int/publications-detail/clinicalmanagement-of-severe-acute-respiratory-infection-whennovel-coronavirus-(ncov)-infection-is-suspected Accessed May 14, 2020.

17. Davanzo R, Moro G, Sandri F, Agosti M, Moretti C, Mosca F. Breastfeeding and Coronavirus Disease-2019. Ad interim indications of the Italian Society of Neonatology endorsed by the Union of European Neonatal \& Perinatal Societies. Matern Child Nutr. 2020:e13010.

18. Furlow B. US NICUs and donor milk banks brace for COVID-19. Lancet Child Adolesc Health. 2020; 4:355.

19. Van Doremalen N, Bushmaker R, Karesh W B, Munster V J. Stability of Middle-East respiratory syndrome coronavirus in milk. Emerg Infect Dis. 2014; 20:1263-4.

20. Rabenau HF, Cinatl J, Morgenstern B, Bauer G, Presier W, Doerr HW. Stability and inactivation of SARS corona virus. Med Microbiol Immunol. 2005; 194:1-6.

21. Chin AWH, Chu JTS, Perera MRA, Hui KYH, Yen HL, Chan MCW, et al. Stability of SARS-CoV-2 in different environmental conditions. Lancet Microb. 2020;1:E10.

22. Chambers CD, Krogstad P, Bertrand K, Contreras D, Tobi $\mathrm{NH}$, Bode L, et al. Evaluation of SARS-CoV-2 in breastmilk from 18 infected women. Preprint. medRxiv. 2020;2020.06.12.20127944. Published 2020 Jun 16.

23. Conzelmann C, Gro R, Meister TL, Todt D, Krawczyk A, Dittmer U, et al. Holder pasteurization inactivates SARSCoV-1 2 in human breast milk. Preprint. bioRxiv. 2020.06.17.155689. 